\title{
Partial detachment of high power discharges in ASDEX Upgrade
}

A. Kallenbach ${ }^{1}$, M. Bernert ${ }^{1}$, M. Beurskens ${ }^{2}$, L. Casali ${ }^{1}$, M. Dunne ${ }^{1}$, T. Eich $^{1}$, L. Giannone ${ }^{1}$, A. Herrmann ${ }^{1}$, M. Maraschek ${ }^{1}$, S. Potzel ${ }^{1}$, F.

Reimold $^{1}$, V. Rohde ${ }^{1}$, J. Schweinzer ${ }^{1}$, E. Viezzer ${ }^{1}$, M. Wischmeier ${ }^{1}$, ASDEX Upgrade Team

${ }^{1}$ Max Planck Institute for Plasma Physics, Garching, GERMANY

${ }^{2}$ CCFE, Culham Science Centre, Abingdon, OX14 3DB, UK

E-mail: Arne.Kallenbach@ipp.mpg.de

\begin{abstract}
Detachment of high power discharges is obtained in ASDEX Upgrade by simultaneous feedback control of core radiation and divertor radiation or thermoelectric currents by the injection of radiating impurities. So far $2 / 3$ of the ITER normalized heatflux $\mathrm{P}_{\text {sep }} / \mathrm{R}=15 \mathrm{MW} / \mathrm{m}$ has been obtained in ASDEX Upgrade under partially detached conditions with a peak target heatflux well below $10 \mathrm{MW} / \mathrm{m}^{2}$. When the detachment is further pronounced towards lower peak heatflux at the target, substantial changes in ELM behaviour, density and radiation distribution occur. The time-averaged peak heat flux at both divertor targets can be reduced below $2 \mathrm{MW} / \mathrm{m}^{2}$, which offers an attractive DEMO divertor scenario with potential for simpler and cheaper technical solutions. Generally, pronounced detachment leads to a pedestal and core density rise by about $20-40 \%$, moderate $(<20 \%)$ confinement degradation and a reduction of ELM size. For AUG conditions, some operational challenges occur, like the density cut-off limit for X-2 ECRH heating, which is used for central tungsten control.
\end{abstract}




\section{Introduction}

To ensure a sufficient lifetime of the divertor in ITER and DEMO, operation under at least partial detachment (PD) will be mandatory [1] [2]. PD is defined as a significant reduction of heat flux and pressure along field lines between midplane and divertor target for the first few $(\approx 2)$ power decay lengths in the scrape-off layer $(\mathrm{SOL})$. PD of the outer divertor is obtained by radiative cooling of AUG high power discharges $\left(\mathrm{P}_{\text {heat }} \approx 20 \mathrm{MW}\right)$, in combination with high divertor neutral deuterium densities. Since the inner divertor exhibits more prominent detachment compared to the outer divertor under standard conditions, the outer divertor is considered as the critical location.

The qualitative behaviour of the power load distribution along the outer target is sketched in figure 1, where also terms for different detachment states are introduced. For attached

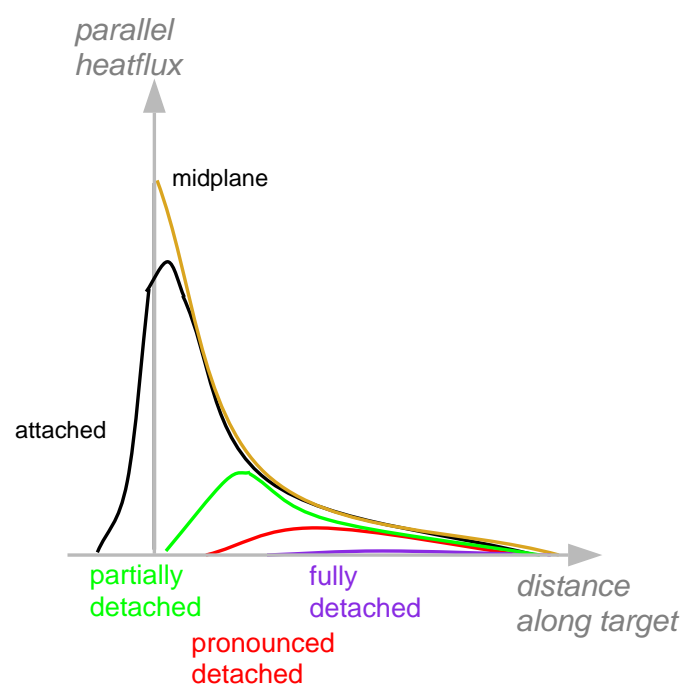

Figure 1. Qualitative sketch of target heatflux profiles for different detachment states.

conditions, the width $\lambda_{\text {int }}$ of the power load profile has been parametrized as the combination of an exponential decay width, $\lambda_{q}$, and a broadening parameter, $S, \lambda_{\text {int }} \approx \lambda_{q}+1.64 S$ [3]. $\lambda_{q}$ is understood as the power width at the divertor entrance, $\mathrm{S}$ describes the Gaussian shaped power spreading. Typical AUG values $\left(\mathrm{I}_{p}=1 \mathrm{MA}\right)$ are $\lambda_{q}=2 \mathrm{~mm}, \mathrm{~S}=1.5 \mathrm{~mm}$ and $\lambda_{\text {int }}=4.5 \mathrm{~mm}$, mapped to the outer midplane [4] [5]. Taking into account the flux expansion $\mathrm{f}_{x} \approx 5$, values of $\lambda_{\text {int }}=2-2.5 \mathrm{~cm}$ are typical along the attached outer target. Both $\lambda_{q}$ and $\mathrm{S}$ were found to scale with inverse poloidal field $\propto 1 / \mathrm{B}_{p}$ [6]. The broadening $\mathrm{S}$ increases strongly towards low divertor temperatures [7]. The trend of increasing $S$ for lower divertor temperature continues towards detachment, but with the onset of strong atomic physics processes during PD below 
$\mathrm{T}_{e}=5-10 \mathrm{eV}$ a more complicated spatial distribution beyond simple power spreading is expected. An outward shift of the peak power flux is observed under these conditions.

Partial detachment starts at the strike point for standard vertical divertor geometry and evolves towards the SOL. This paper investigates the variation of the degree of partial detachment in ASDEX Upgrade high power discharges and its effect on main plasma parameters. The degree of the detachment is extended to even higher values than required for ITER, resulting in pronounced detachment over several power widths and a very low peak power load. Still, the outer SOL wing in the divertor remains attached, which is regarded as important for the sustainment of good (i.e. factor 200) divertor neutral plugging against the main chamber. The good neutral divertor compression is expected to be strongly reduced for full detachment along the whole target, when the neutral plugging by the plasma in the far divertor wing is lost. Assuming a similar power width in AUG compared to ITER [5] and adopting similar Greenwald and separatrix densities, the AUG divertor conditions are expected to approach those expected for ITER in absolute terms of temperature, density and neutral pressure for high values of the normalized separatrix power flux, $\mathrm{P}_{\text {sep }} / \mathrm{R}$.

This paper is organized as follows. Feedback control of discharges with high power flux into the divertor (high $\mathrm{P}_{\text {sep }}$ ) and partial detachment with nitrogen seeding is introduced in section 2. Section 3 describes the plasma behavior when the detachment is further pronounced towards even lower peak power loads. The parameter range of AUG discharges with different degrees of detachment is summarized in section 4 , and a qualifier for the detachment state is introduced which is based on engineering parameters. Some physics details of the detachment process are discussed in section 5, and finally conclusions are drawn.

\section{Feedback control of high divertor power fluxes}

A versatile double feedback system is available in AUG which allows independent control of divertor (by $\mathrm{N}$ ) and core (by $\mathrm{Ar}$ or $\mathrm{Kr}$ ) radiation [8]. For divertor power exhaust studies, low core radiation levels are helpful for the maximisation of $\mathrm{P}_{\text {sep }}$ and thus just one low-Z impurity is used for seeding. A thermoelectric current sensor is used to control the target heatflux, where the effect of ELMs is removed by an asymmetric median real time filter [9]. Since its signal quite closely follows the electron temperature near the strike point for attached conditions, it has been scaled to match typical $\mathrm{T}_{e}$ values and labelled Tdiv (500 ampere total thermoelectric current correspond to Tdiv=10"eV"). Figure 2 shows a discharge 


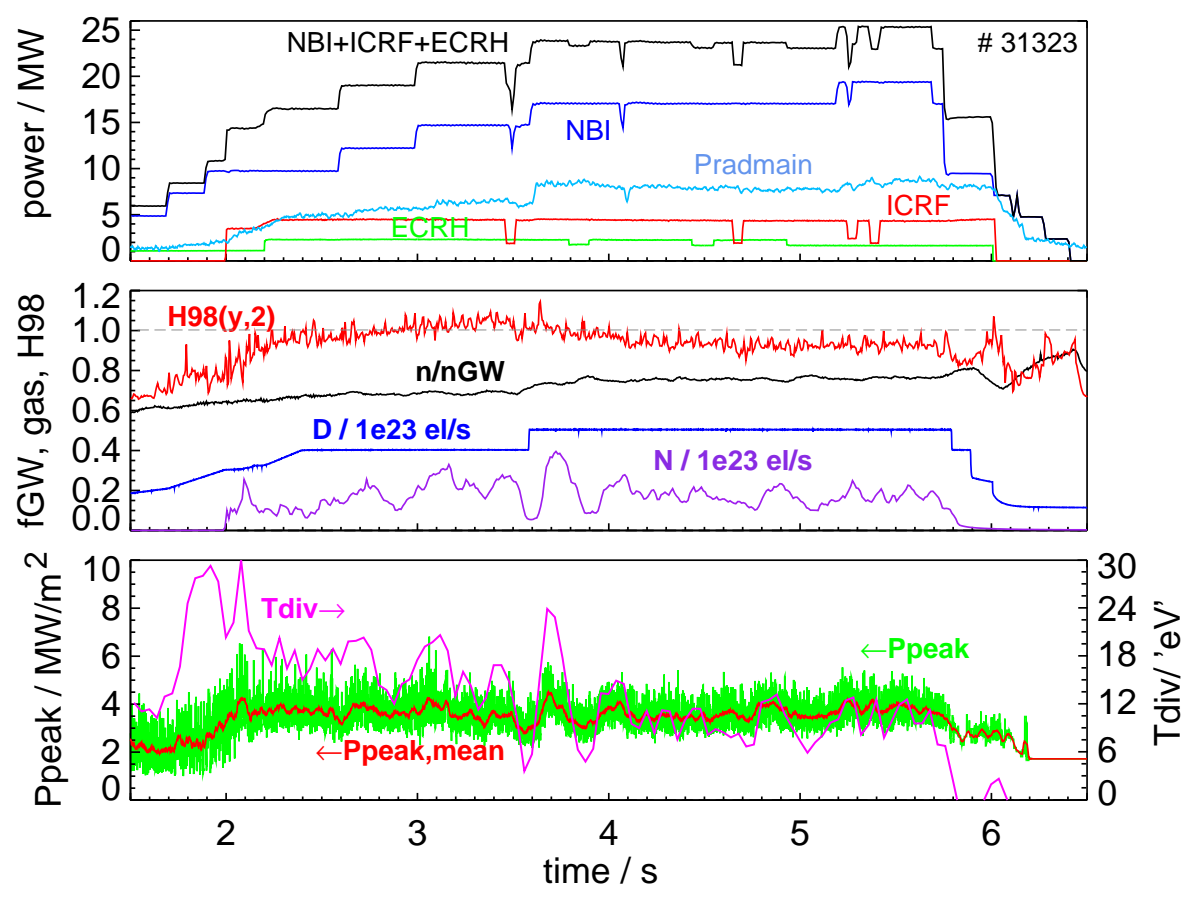

Figure 2. Time traces of an $A U G$ discharge with high $P_{\text {sep }} / R$ up to $10 \mathrm{MW} / \mathrm{m}$ and a target peak power load around $5 \mathrm{MW} / \mathrm{m}^{2}$. The partially detached conditions are obtained by high deuterium puffing, 1/3 of the nominal cryo-pumping speed and Tdiv feedback control using nitrogen divertor puffing. Nitrogen core concentration $c_{N} \leq 1.5 \%, \beta_{N} \approx 3, I_{p}=1 M A, q_{95}=$ 4.3 , inner divertor neutral pressure $P_{0}=4 \mathrm{~Pa}$. Major radius $R=1.65 \mathrm{~m}$.

with up to $25 \mathrm{MW}$ heating power and divertor power dissipation by nitrogen seeding and a high deuterium pressure. Feedback control is done with the nitrogen gas puff as actuator using the thermoelectric Tdiv as sensor, starting at $\mathrm{t}=2 \mathrm{~s}$ with a set value of $\mathrm{Tdiv}=20 \mathrm{eV}$ which is ramped down to $10 \mathrm{eV}$ at $3 \mathrm{~s}$, this set value is kept till $\mathrm{t}=5.8 \mathrm{~s}$. Deuterium is puffed in feedforward, as shown in figure 2. Stable plasma parameters are achieved under these partially detached conditions. $\mathrm{P}_{\text {sep }} / \mathrm{R}=10 \mathrm{MW} / \mathrm{m}$, about $2 / 3$ of the ITER value of $15 \mathrm{MW} / \mathrm{m}$ have been reached with a time averaged peak heatflux at the outer target of about $5 \mathrm{MW} / \mathrm{m}^{2}$. $\mathrm{P}_{\text {sep }}$ is calculated as the difference of the absorbed heating power and the main chamber radiation calculated in real time (a detailed description is given in [8]) as shown in the traces of figure 2. The main chamber radiation includes also the small contribution of the main chamber scrapeoff layer radiation, which cannot be separated reliably by deconvolution from the confined plasma radiation due to the steep gradients at the separatrix. Without any power dissipation, about $45 \mathrm{MW} / \mathrm{m}^{2}$ would be expected from the attached heat load width scaling [5]. The measurements indicate partial detachment, with a moderate midplane-target pressure drop of 
about a factor 3 .

High power discharges also approach the beta limit. Nitrogen seeding, primarily used for enhancement of the divertor radiation, also causes a confinement improvement [10], which is counteracted by the degrading effect of strong deuterium puffing / divertor recycling. The discharge shown in figure 2 has a $\beta_{N}$ of 3 and strong MHD activity which is dominated by fishbones. A slight reduction of $\mathrm{H}_{98}$ from 1 to 0.95 around $3.7 \mathrm{~s}$ may be caused by increased MHD activity as $\beta_{N}$ exceeded the values of 3 or by the beginning detachment. Despite operation with reduced cryo pumping speed to $1 / 3$ of the AUG standard setting using a newly installed cryogenic Helium valve, the foreseen ITER neutral pressure limit of $10 \mathrm{~Pa}$ [11] could not be reached at maximum gas injection. AUG operation with turbo-pumps alone is planned in the near future for a further increase of the neutral pressure.

In the following, experiments are discussed which aim towards a more pronounced divertor detachment, facilitating strong momentum loss along field lines in the divertor. Such conditions relax the challenges of a DEMO divertor design, allowing its operation with a simpler standard vertical target configuration.

\section{Transition towards full detachment}

Time traces of a discharge entering pronounced detachment are shown in figure 3, where almost complete detachment of the divertor has been obtained by the combination of constant $\mathrm{D}$ and $\mathrm{N}$ puff levels. The achievement of pronounced detachment of the outer divertor is indicated by the zero-crossing of the Tdiv signal [9]. The evolution towards full detachment after $3 \mathrm{~s}$ is accompanied by a rise of the plasma density to almost the Greenwald value and a reduction of the $\mathrm{H}$-factor to 0.85 . Pedestal profiles are shown in figure 4 for the 3 time points indicated. The substantial temperature drop during pronounced detachment is almost compensated by the density rise, resulting in a pressure drop of about $15 \%$ at $\rho_{\text {pol }}=0.8$, the same relative pressure drop is observed in the plasma center.

After $\mathrm{t}=5 \mathrm{~s}$, the performance of \# 30506 is slightly further degraded possibly due to mild central tungsten accumulation (see figure 7b) developing after the trip of central ECRH, which had to be used in low single-pass absorption O-2 mode since the density exceeds the X-2 cut-off value for AUG conditions. Plasma performance recovers after an additional NBI source is switched on at $5.5 \mathrm{~s}$. The plasma $\bar{Z}_{e f f}$ is constant with a value of 2 after $2.8 \mathrm{~s}, \mathrm{~N}$ is its main impurity contributor with a concentration of $2 \%$ from CXRS measurements. Plasma 

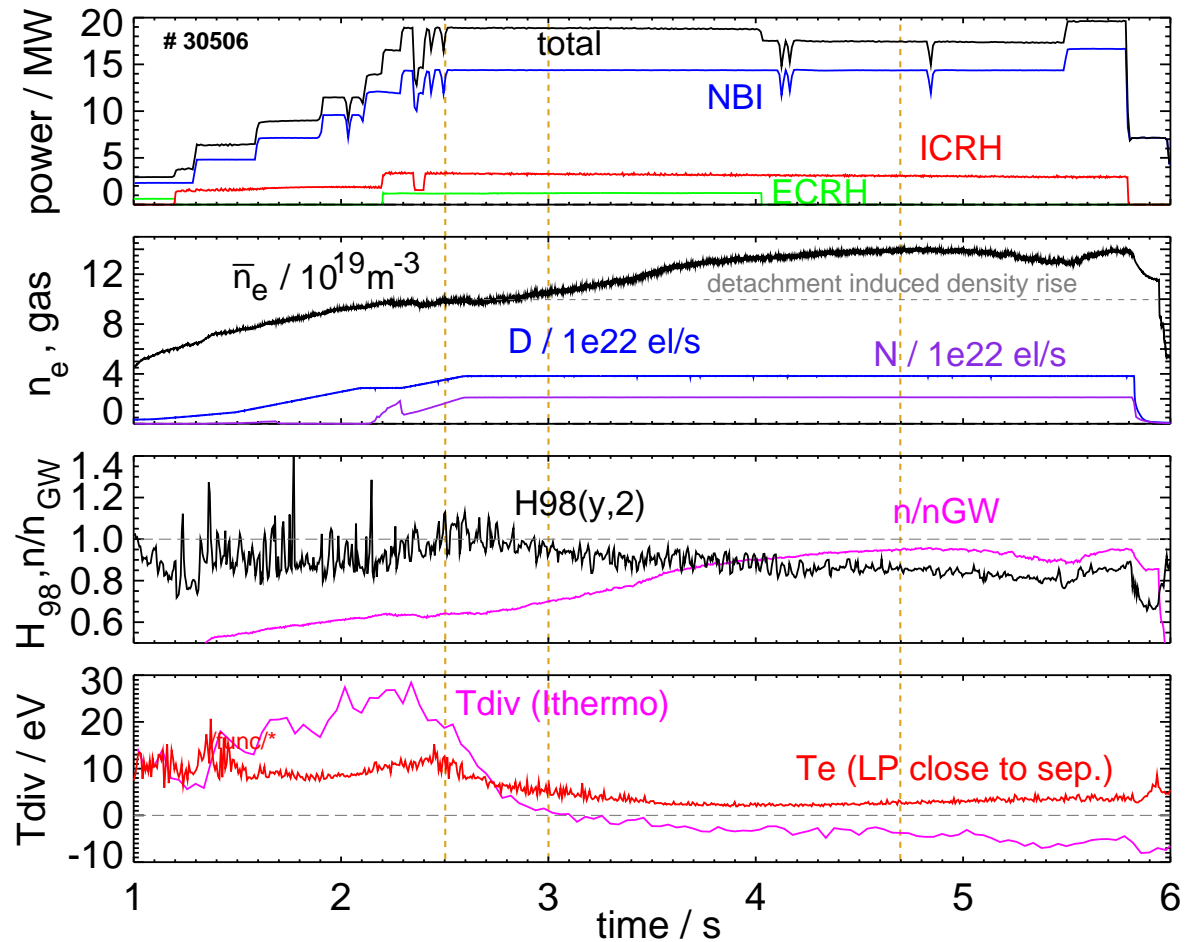

Figure 3. Time traces of a high power AUG discharge with strong feed-forward D puff and $N$ seeding. At $t=3 \mathrm{~s}$, pronounced detachment is entered, in the following the detached region expands further along the outer target and a rise in line-averaged density occurs. $I_{p}=1.2 \mathrm{MA}$, $q_{95}=3.7, \delta_{u p}=0.1, \delta_{l o w}=0.4$. Divertor neutral pressure $P_{0}=3.4 \mathrm{~Pa}$, only $1 / 3$ of the cryo pump activated. Dotted vertical lines denote times where divertor profiles from Langmuir probes are shown in figure 5.

parameters along the outer target are shown for 3 different times of figure 3 in figure 5 . The peak heat flux measured my the Langmuir probes reduces to values far below $1 \mathrm{MW} / \mathrm{m}^{2}$. IR thermography, which measures also the heat load by radiation, stays below $2 \mathrm{MW} / \mathrm{m}^{2}$ (not shown in figure). Tdiv crossing zero marks the transition from partial to pronounced detachment, with substantial reduction of pressure and heat flux around the strike point. It is a general observation for such AUG conditions that a reduction of $\mathrm{T}_{e}$ in front of the target is connected to a drop in electron pressure, similar to results reported from ALCATOR C-Mod [12]. During pronounced detachment, heat flux and pressure are strongly reduced over about $10 \mathrm{~cm}$ or several power decay lengths. For reference, the (attached) power width inferred from the Eich scaling [5] is $\lambda_{\text {int }} \approx 2 \mathrm{~cm}$ along the target for this discharge. In the divertor far SOL, the $\mathrm{j}_{\text {sat }}$ and $\mathrm{T}_{e}$ profiles are hardly effected by the detachment at distances more than $10-15 \mathrm{~cm}$ away from the strike point (fig. 5). Consequently, the outer divertor is not fully detached, and 


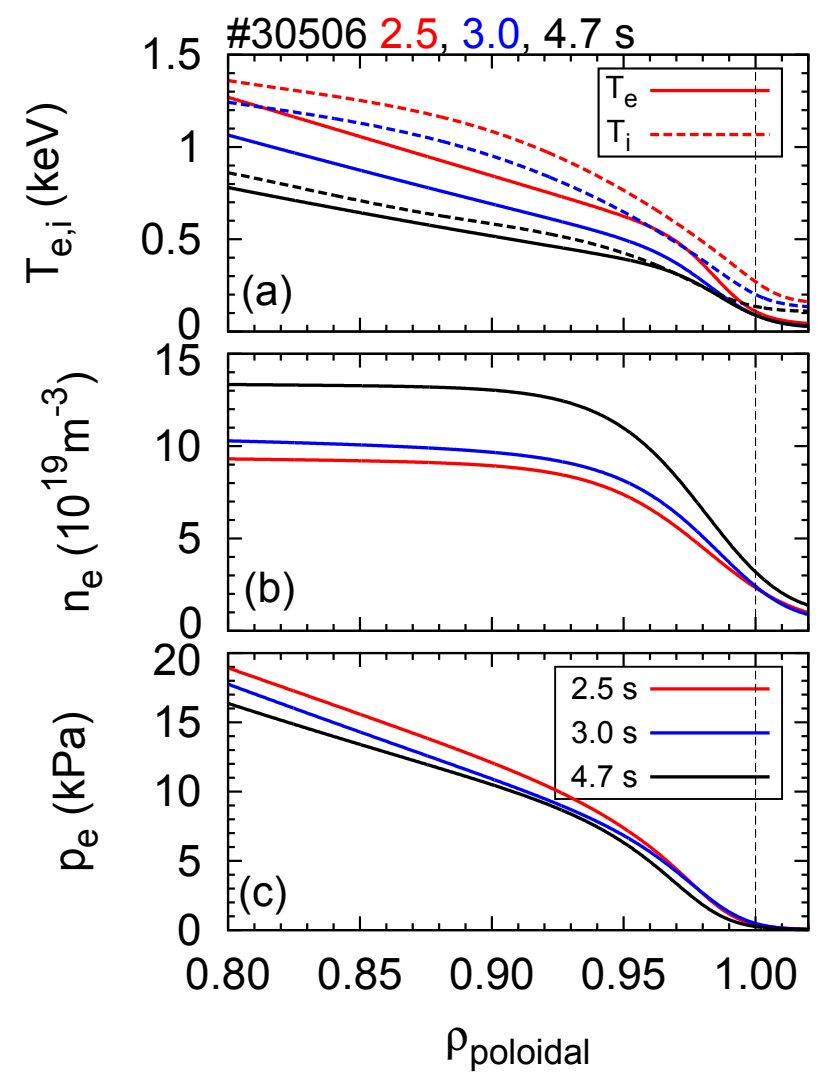

Figure 4. ELM averaged pedestal profiles from fits to Thomson scattering $\left(n_{e}, T_{e}, p_{e}\right)$ and CXRS $\left(T_{i}\right)$ for the time points indicated in figure 3.

the term pronounced detachment is used, which emphasizes that a good neutral plugging by the divertor is still present.

Tdiv appears as a highly suited sensor for detachment monitoring and control. As shown in figure 5, Tdiv approaches small values during partial detachment, negative values of Tdiv are a robust indicator of pronounced detachment over the full AUG parameter range of plasma current, power and densities. However, the control algorithm requires further improvement and adaption to cope with the low dynamic range of Tdiv during detachment and the appearance of negative values, which can no longer be associated to a power flux or temperature. Tdiv $=0$ is associated with a vanishing thermoelectric current. At this stage, the Pfirsch-Schlüter components of the passive current may still remain [13], but cancel to zero along the tile. The slightly negative values of Tdiv occuring during pronounced detachment are supposed to be caused either by thermoelectric currents in the far divertor SOL connected to in-vessel components, where the divertor acts as the cold end, or by the negative PfirschSchlüter currents in the private flux region. The negative values of Tdiv occur very robustly 

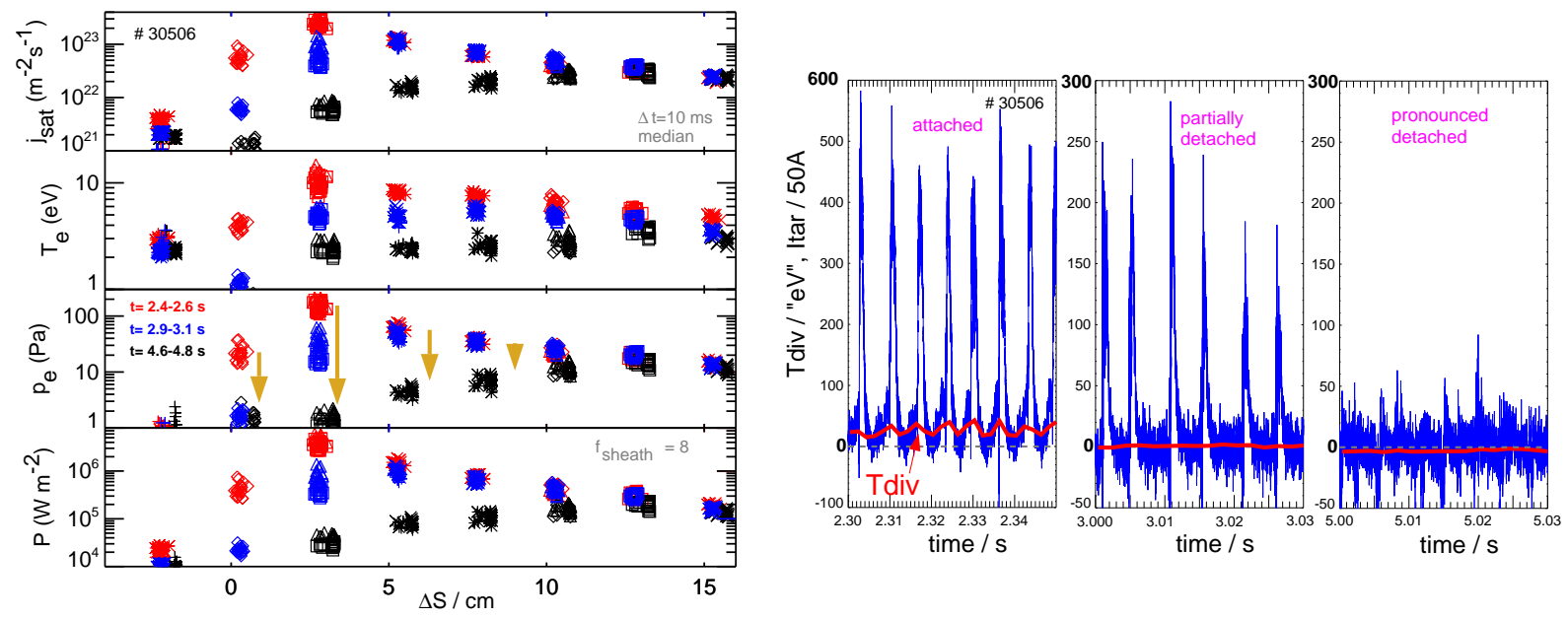

Figure 5. left: Divertor profiles from Langmuir probes for the attached, partially detached and pronounced detached conditions. Profiles are median-filtered and time-averaged over $200 \mathrm{ms,}$ removing the contribution of ELMs as far as the limited time resolution permits. right: Tdiv as derived from the passive current measurement by means of a shunt in an outer target tile. Tdiv has been defined as the total inter-ELM current between outer and inner divertor divided by 50. The high time resolution $(0.2 \mathrm{MHz})$ of the passive current measurement and its high $S / N$ ratio make it an ideal real time sensor. The removal of ELM signatures is done by an asymmetric median filter in LabView.

over a broad experimental parameter range. While the detachment proceeds along the outer target, the divertor radiation develops a strong radiation zone inside the confined plasma at the X-point region [14]. Finally during the most pronounced detachment phase after $t=4 \mathrm{~s}$, the divertor radiation is dominated by the emission inside the X-point, as shown in figure 6 . This sugggests the existence of parallel gradients of $\mathrm{T}_{e}$ towards the outer midplane, similar to a MARFE. Even during this stage, the outer divertor is not completely detached as can be seen from the Langmuir probe profiles in figure 5.

In the DIII-D tokamak, divertor electron temperature and density profiles could be measured by divertor Thomson scattering during H-mode detachment experiments using D puffing [15]. A cold $\left(\mathrm{T}_{e}<5 \mathrm{eV}\right)$ and dense region is found to encompass the X-point region during detached divertor conditions, with a steep parallel gradient region following upstream and a loss of pressure balance along the field lines. If detachment proceeds, the radiation moves into the $\mathrm{X}$-point region and confinement degradation may occur depending on connection length to the midplane and plasma parameters.

Figure 7 shows the impurity evolution during pronounced detachment. The additional 

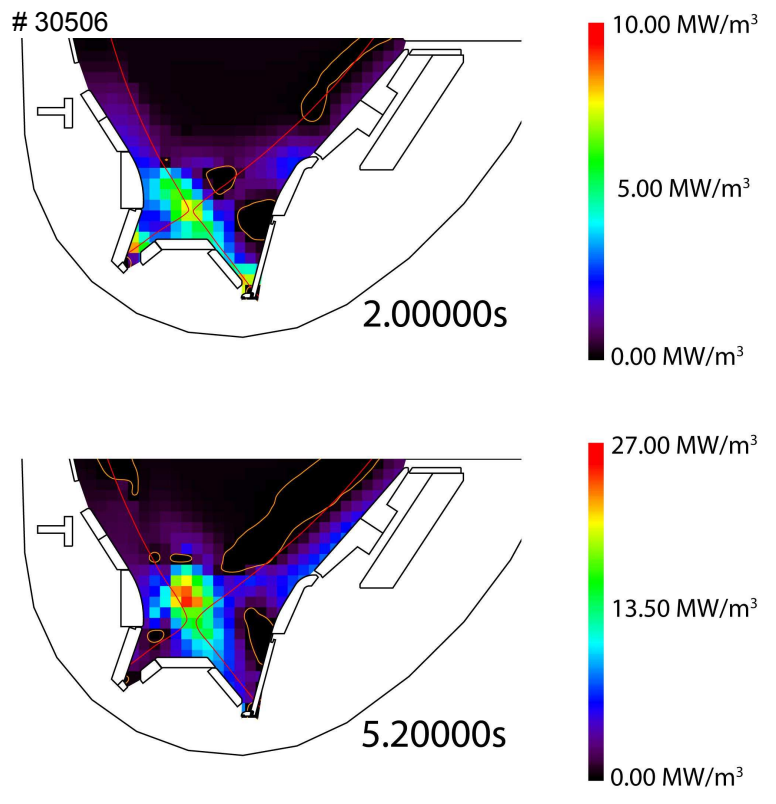

Figure 6. Tomographic reconstruction for the attached and detached phases of the discharge in figure 3. During pronounced detachment, a radiating zone inside the confined plasma at the $X$-point has clearly developed.
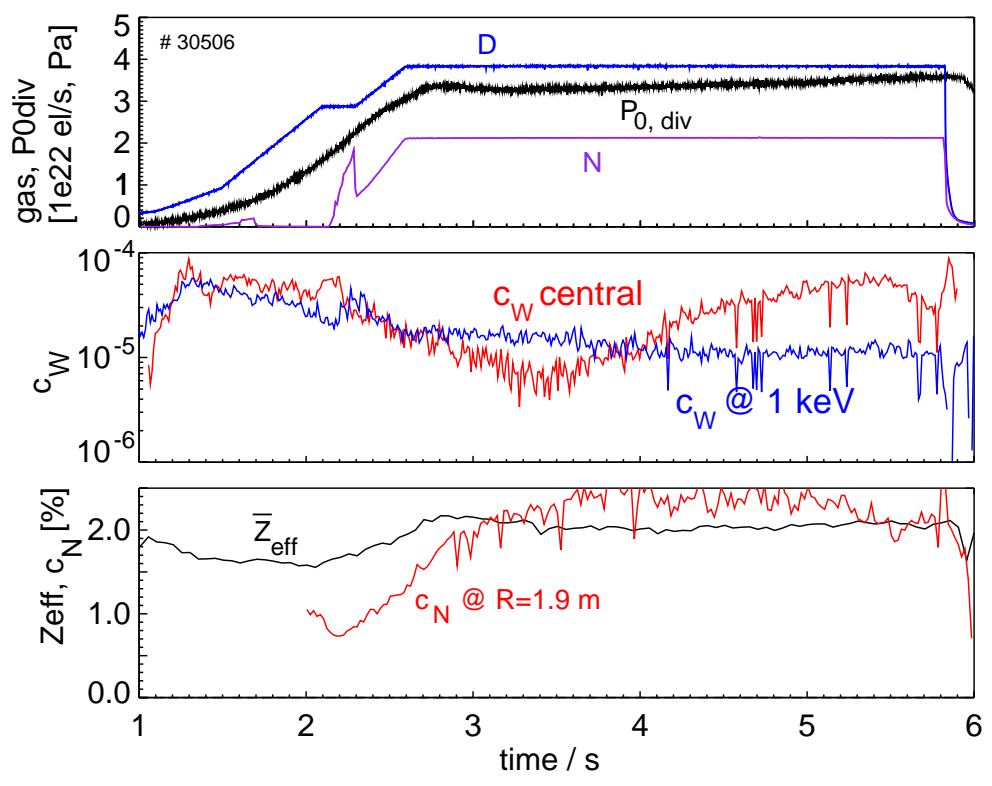

Figure 7. Time traces of impurity behaviour of the discharges introduced in figure 3. The pronounced detachment entered after $t=3 s$ is not connected to a drastic increase of the nitrogen content. ECRH is tripped by a sniffer probe at $4 \mathrm{~s}$ due to stray radiation caused by low $O-2$ absorption.

impurity level required to proceed from partially detached conditions before $t=3 \mathrm{~s}$ to the pronounced detachment is moderate. Some central peaking of the tungsten concentration 
is observed after the trip of the ECRH at $4 \mathrm{~s}$. The density rise during pronounced detachment hampers the use of ECRH in AUG, since the central density exceeds the X-2 cut-off value. The observed slow time evolutions of plasma parameters call for improved control schemes.

\section{Parameter space for radiative cooling and detachment in ASDEX Upgrade}

Detachment of H-mode discharges can be invoked by low-Z impurity seeding, a rise of the divertor neutral pressure due to $\mathrm{D}$ puffing or a reduction of the separatrix power flux $\mathrm{P}_{\text {sep }}$ by core radiator seeding. The interplay of these mechanisms can be complicated and non-linear. A rise of the neutral pressure generally supports divertor detachment, but may be insufficient as sole measure [14] [16]. The use of impurities supports detachment by radiation, and causes much less confinement degradation or even an improvement. Best plasma performance at benign divertor loads is obtained by a combination of a high neutral pressure and impurity seeding.

Figure 8 shows the parameter range of a database of AUG high power discharges with different core radiation levels and $\mathrm{P}_{\text {sep }}$ values. Many of the discharges have nitrogen seeding, high core radiation levels were induced by Ar or Kr seeding, partly combined with divertor $\mathrm{N}$ seeding. Red symbols denote pronounced detachment with peak power loads from thermography below $2 \mathrm{MW} / \mathrm{m}^{2}$ and green symbols discharges close to this condition, with power loads below about $5 \mathrm{MW} / \mathrm{m}^{2}$ for discharges with high heating power.

An interesting question is the dependence of the onset of divertor detachment as a function of the main players $\mathrm{P}_{\text {sep }}$ and the divertor neutral pressure of $\mathrm{D}$ and $\mathrm{N}, \mathrm{p}_{0}$ and $\mathrm{p}_{0, N}$. Although a non-linear and complicated dependence of divertor detachment on these parameters has to be expected, a reasonable prediction of pronounced detachment onset has been obtained in AUG, as shown in figure 9. Pronounced detachment occurs when Tdiv $\leq 0$, the detachment qualifier

$$
q_{\text {det }}=P_{\text {sep }} / R\left(p_{0}+18 p_{0, N}\right)^{-1} \times 1.3 \mathrm{~Pa} \mathrm{~m} / \mathrm{MW}
$$

is reduced below the value of 1 for these conditions. Expression 1 was obtained by leastsquare fitting of non-negative functions of Tdiv versus $\mathrm{P}_{\text {sep }} / \mathrm{R}$ and the weighted pressures, the result was normalized to obtain $\mathrm{q}_{d e t}=1$ for $\mathrm{Tdiv}=0$. This formula is currently only valid for ASDEX Upgrade. Only cases with nitrogen seeding have been considered to have one dominant divertor radiating impurity. Since no direct pressure measurement for nitrogen in 

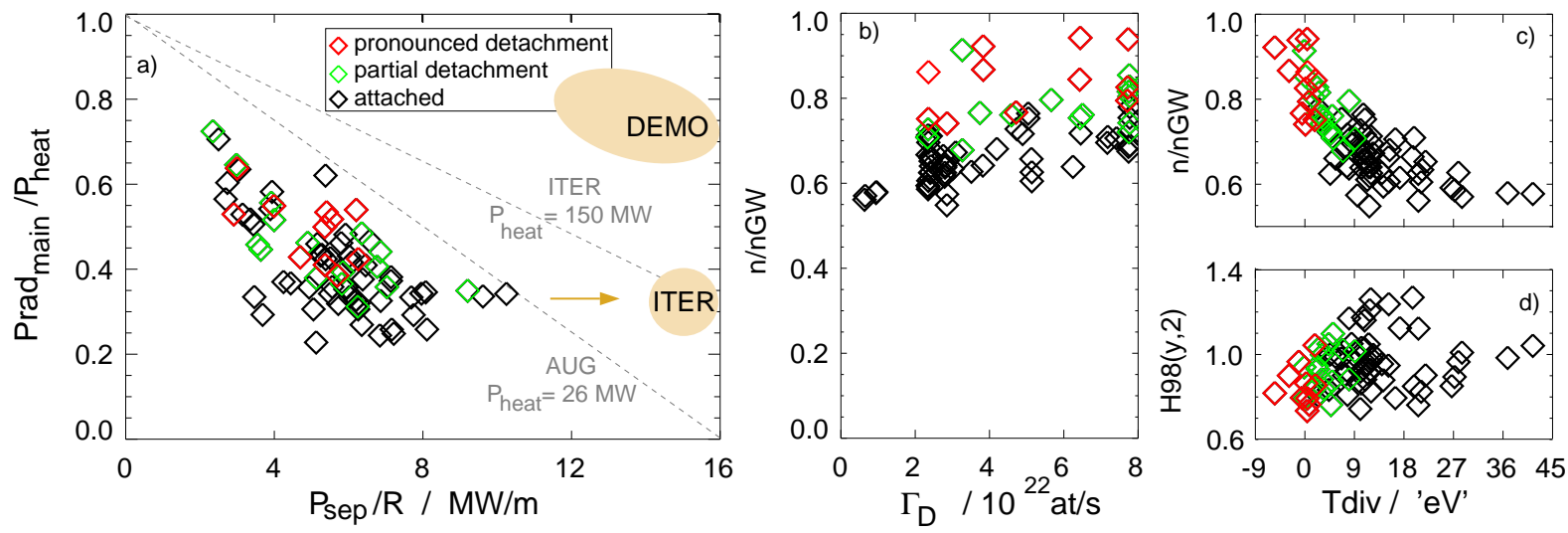

Figure 8. a) Operational diagram of core radiation and divertor power flux in ASDEX Upgrade and envisaged parameters for ITER and DEMO. Each point represents a quasistationary discharge phase of at least 100 ms regarding gas puff rates and stored energy, symbols denote different detachment states. Lines represent the possible parameter space for a given $P_{\text {sep }} / R$. Main chamber and divertor radiated powers are taken from the real time evaluation [8]. b) Greenwald fraction versus divertor neutral pressure below the roof baffle. c) Greenwald fraction vs. Tdiv. d) $H_{98}$ vs. Tdiv.

the divertor is available, its pressure has been derived from the measured total pressure and the relative $\mathrm{N}$ and $\mathrm{D}$ valve fluxes for quasi-stationary conditions. The factor 18 in $q_{\text {det }}$ represents the much stronger detachment inducing capability of $\mathrm{N}$ by radiation in comparison to $\mathrm{D}$.

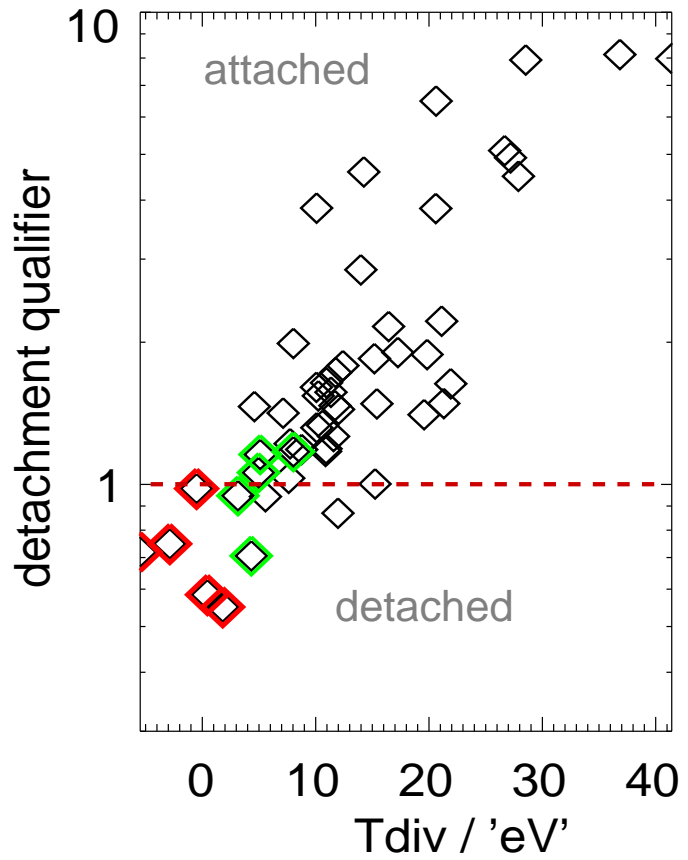

Figure 9. Detachment qualifier vs. Tdiv for the shots with D fueling or dominant $N$ seeding. 
The detachment qualifier eq. 1 must not be confused with the degree of detachment (DoD) introduced by Loarte [17]. The latter measures the pressure drop by a deviation of $j_{\text {sat }}$ from the expected $\mathrm{n}_{e}^{2}$ scaling. This is usually applied under L-mode conditions and requires density ramps or reference phases. The detachment qualifier has been set up for $\mathrm{H}$-mode conditions and includes the effect of impurity seeding. Since it is based on mainly engineering parameters (heating power, gas valve fluxes, neutral pressure from pumping speed), it has - in contrast to DoD - a predictive capability and could be used for discharge planning and control.

\section{Detachment process and origin of the density rise}

During divertor detachment a substantial increase of pedestal and line-averaged electron densities occurs. As can be seen in figure $8 \mathrm{~b}$ and $\mathrm{c}$ by the strong correlation of the Greenwald fraction with Tdiv, the density rise is not primarily caused by the deuterium gas puff or recycling. For different fueling levels, occurence of detachment leads to a substantial increase of the line-averaged density. Its close correlation with the parameter Tdiv suggests an effect connected to the divertor temperature. To demonstrate this for constant D fueling conditions, figure 10 shows time traces of a high power discharge with double $(\mathrm{N}, \mathrm{Ar})$ radiative feedback where detachment has been invoked by requesting a higher core radiation level by Ar seeding at constant $\mathrm{D}$ fueling. The additional core radiation causes partial detachment because of the reduction of $\mathrm{P}_{\text {sep }}$, the $\mathrm{PD}$ extends then towards pronounced detachment before the radiation is reduced again after $t=4 \mathrm{~s}$. The density rise appears very similar to the cases with $\mathrm{N}$ divertor radiation induced detachment. Divertor parameters for the discharge of figure 10a are shown in figure $10 \mathrm{~b}$, the qualitative behaviour appears quite similar to those in figure 5. The detachment appears more prominent in the Tdiv signal as compared to the LP or IR measurement. This is caused by the higher temporal resolution of the underling passive current measurement, which allows a better ELM removal by the median filter.

High density regions occur in both divertors during conditions of high power flux into the divertor and sufficiently high neutral density [18]. Figure 11 shows time traces of these features for the the discharge shown in figure 10. Electron densities up to $10^{21} \mathrm{~m}^{-3}$ are measured during attached conditions, they decrease when the power flux into the divertor is reduced by the Argon seed radiation. Simultaneously, the neutral pressure at the inner divertor entrance measured by an ionization gauge is reduced during the strong Ar seeding. Both coreand divertor seed radiation counter-act against the development of the high density regions, 

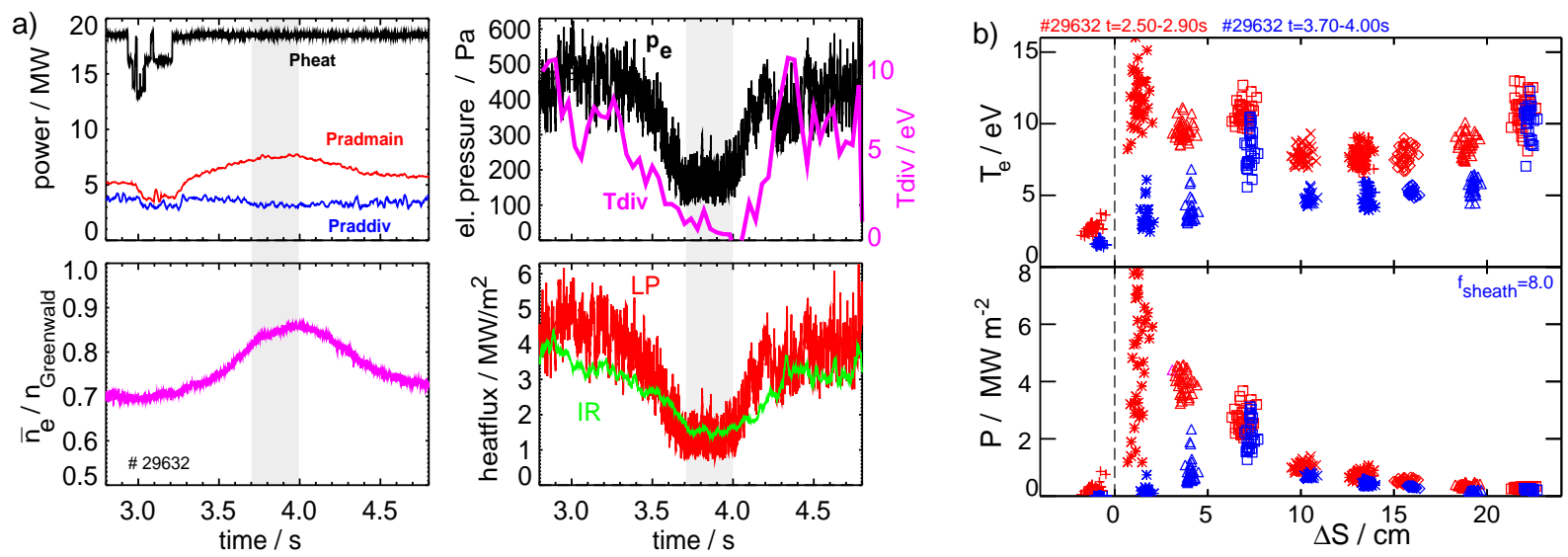

Figure 10. a) Time traces of a high power AUG discharge with strong D puff and N+Ar seeding. Partial detachment between $t=3.6-4.2 s$ is obtained by requesting higher core (Ar) radiation, causing a drop in heatflux and electron pressure in the outer SOL near the strike point. $P_{e}, L P$ and IR are ELM averaged, while ELMs are removed from Tdiv by a numerical filter. The ELM frequency stays roughly constant over the detachment cycle. b) Divertor profiles from Langmuir probes for the attached and detached conditions.

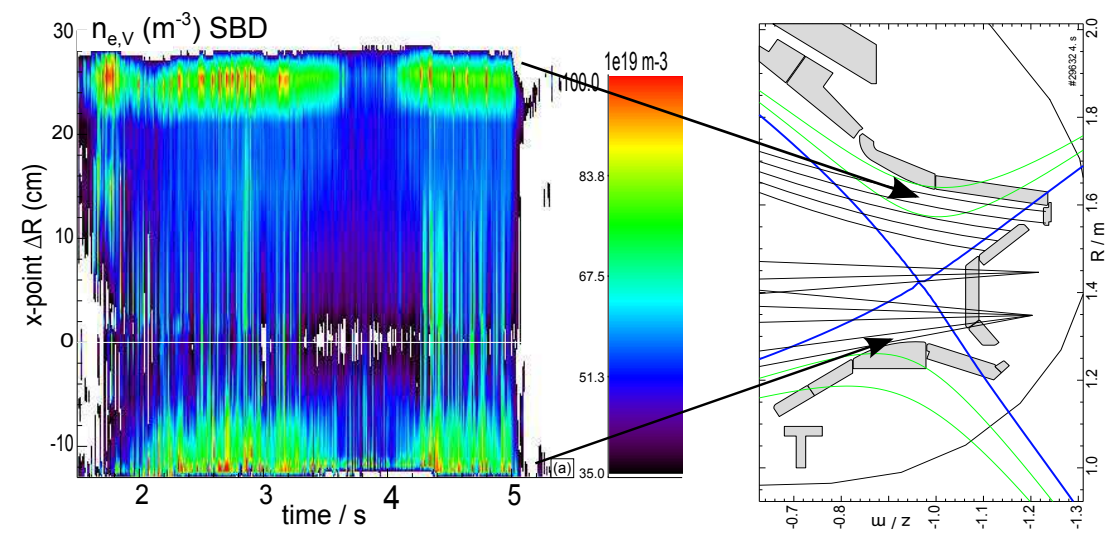

Figure 11. a) Divertor densities measured by Stark broadening of deuterium Balmer lines and geometry of the viewing lines for the discharge shown in figures 10. Regions of high electron density are observed in both divertors. Their densities decrease during the phase of reduced divertor power flux caused by the Argon seeding.

the increased radiation has a similar effect compared to a reduction of the heating power [19]. A possible explanation for the plasma density rise observed under these conditions is better neutral fuelling in the absence of the high density structures in the SOL [14]. When the power flux into the divertor is further reduced, a strong radiating zone develops inside the X-point (see figure 6).

The divertor detachment is reversible, as seen in figure 10, but its stationary control is 
challenging due to global parameter changes during simultaneous core and divertor control. The changes regard both ELM behaviour and the plasma density. Figure 12 shows the effect of a rise in core radiation for two different experimental conditions. Seeding of Ar or $\mathrm{Kr}$ at low to moderate divertor pressure (below $\approx 3 \mathrm{~Pa}$ ) and attached conditions reduces the type-I ELM frequency due to the reduction of the heat flux through the pedestal, and a rise of pedestal and line-averaged density of $15-20 \%$ occurs (left fig. 12). The density rise is related to the prolongation of the recovery time between ELMs under these conditions. At higher neutral pressure (right, discharge of fig. 10), smaller, more benign ELMs occur and no change of the density is observed until detachment is invoked, when the density rises by $15-20 \%$ as well. The reduction of the ELM size is supposed to be caused by the increase of the pedestal collisionality by the combined effects of $\mathrm{Z}_{e f f}$ rise, density rise and temperature reduction [20]. A direct impact of the detachment on the ELM size cannot be excluded and may be masked by the effects of the pedestal parameter changes.
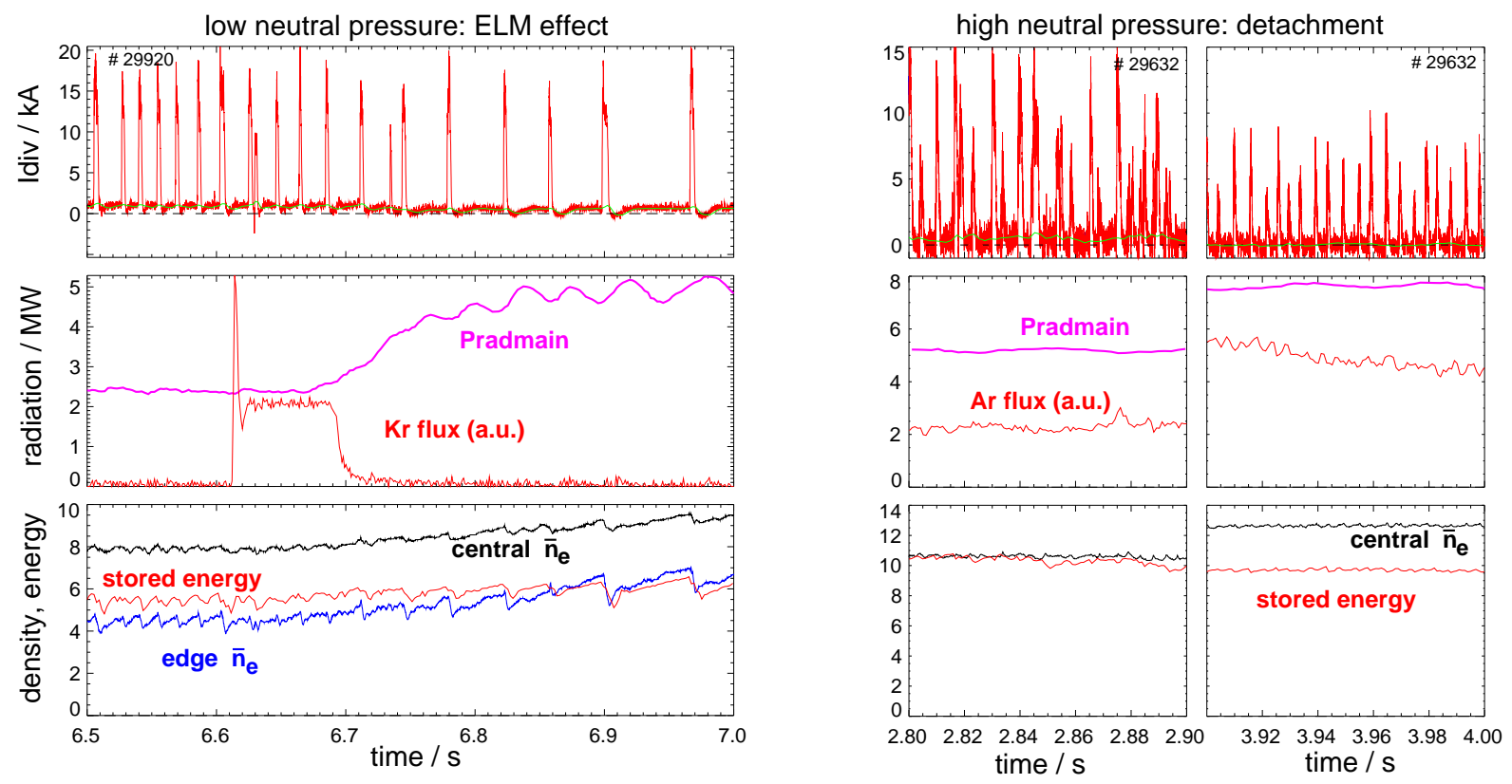

Figure 12. Effect of increased core radiation (Kr/Ar) on ELMs and plasma density for low divertor neutral pressure (left) and high neutral pressure with onset of partial detachment (right). The low pressure discharge (Kr) has $8 \mathrm{MW}$ heating power and $I_{p}=1 \mathrm{MA}$, the high pressure discharge $(A r) 19 M W$ and $I_{p}=1.2 M A$, see also figure 10.

When the detachment is induced by core radiation ( $\mathrm{Ar}, \mathrm{Kr}$ ), the stored energy shows a weak reduction by $\leq 10 \%$. When nitrogen seeding is used for divertor radiation enhancement, confinement improvement due to nitrogen may compensate detachment induced negative 
effects. Detachment caused by an increase of the divertor neutral pressure is accompanied by a considerable confinement degradation, but exhibits lower $\mathrm{Z}_{e f f}$ and dilution values compared to the impurity case. The combination of core seed radiation, divertor seed radiation and divertor neutral pressure may offer flexibility to adjust the optimum working point for a DEMO reactor.

\section{Conclusions}

Different states of divertor detachment have been investigated in high power AUG discharges by the combination of a high divertor neutral pressure and nitrogen seeding. A transition to a pronounced detachment state is observed with very moderate target peak power load $<2$ $\mathrm{MW} / \mathrm{m}^{2}$ (and a much lower plasma power load as measured by Langmuir probes) even at high values of $\mathrm{P}_{\text {sep }}$. The transition from partial to pronounced detachment appears gradual in target heat flux or pressure. Therefore, the vanishing of the thermolelectric current integrated over the outer target is used as transition marker. Pronounced detachment is accompanied by a substantial rise of pedestal and core densities and reduced ELM amplitudes. For high strongly pronounced detachment, a highly radiative region inside the X-point develops. For a future DEMO, operation with pronounced detachment may be advantageous since it allows for a simpler divertor and may assist the achievement of a high density. The required enhancement of neutral pressure and seeding level leads to performance degradation in AUG, where $\mathrm{H}_{98}$ is reduced to values of $0.7-1.1$ for pronounced detachment (see figure 8d) depending on experimental conditions. More work is required to extrapolate this behaviour to DEMO parameters, but intermediate detachment grades with acceptable confinement may well be possible, with the benefit of a simpler technical divertor solution with reduced heat loads. Operation with cold plasma conditions in front of the target plates may also support the achievement of a small or mitigated ELM regime, and will be mandatory for the achievement of an acceptable target erosion rate. A high neutral divertor pressure is also required in AUG during $\mathrm{Ar}$ or $\mathrm{Kr}$ seeding for enhancement of the core radiation. During seeding of $\mathrm{Ar}$ or $\mathrm{Kr}$ at low neutral pressure, hot divertor conditions can lead to a substantial reduction of the type-I ELM frequency, which finally results in core tungsten accumulation.

Comparing to recent JET-ILW results [21] [22], a higher energy confinement in terms of $\mathrm{H}_{98}$ is found in impurity seeded AUG discharges. A possible explanation is the higher beta in AUG high $\mathrm{P}_{\text {sep }} / \mathrm{R}$ discharges, which was found to be quite generally accompanied by higher 
values of $\mathrm{H}_{98}$ [23] [24]. Another difference may lie in the different divertor geometries. AUG (in particular with reduced cryopumping) is typically operated at higher neutral pressure and higher $\mathrm{P}_{\text {sep }} / \mathrm{R}$ compared to JET so far.

In terms of the divertor exhaust parameter $\mathrm{P}_{\text {sep }} / \mathrm{R}, 2 / 3$ of the foreseen ITER value have been realized in AUG under benign divertor conditions with a divertor neutral pressure of $4 \mathrm{~Pa}$. Local divertor parameters are expected to approach those in ITER in absolute values under these conditons. AUG has not hit a limit in $\mathrm{P}_{\text {sep }} / \mathrm{R}$ so far, which could, e.g., be caused by a saturation of divertor radiation due to a shrinking of the radiating zone [25]. The use of nitrogen is limited indirectly by MHD effects as $\beta_{N}$ exceeds the value of 3 in connection with $\mathrm{N}$-induced confinement improvement. Higher values of $\mathrm{P}_{\text {sep }} / \mathrm{R}$ and more pronounced detachment are expected to be achieved with higher divertor neutral pressure, as foreseen for ITER from SOLPS modelling [11]. Some operational challenges appeared in AUG so far, which will not apply to larger devices in this extent. The density rise during pronounced detachment brings the central plasma density above the X-2 cut-off value, and the threat of central tungsten accumulation due to insufficient central heating power density. Here, new ICRF antennas are foreseen to enhance central heating while keeping the tungsten source low.

The Tdiv sensor, based on a simple passive current measurement in the divertor, proved very useful for the detection of divertor detachment. It is expected to be feasible also for dedicated detachment control, which is foreseen for ASDEX Upgrade.

\section{Acknowledgements}

This work has been carried out within the framework of the EUROfusion Consortium and has received funding from the European Union's Horizon 2020 research and innovation programme under grant agreement number 633053. The views and opinions expressed herein do not necessarily reflect those of the European Commission.

[1] LOARTE, A. et al., Phys. Plasmas 18 (2011) 056105.

[2] ZOHM, H. et al., Nucl. Fusion 53 (2013) 073019.

[3] MAKOWSKI, M. et al., Phys. Plasmas 19 (2012) 056122.

[4] EICH, T. et al., Phys. Rev. Lett. 107 (2011) 215001.

[5] EICH, T. et al., Nuclear Fusion 53 (2013) 093031.

[6] SIEGLIN, B. et al., Plasma Physics and Controlled Fusion 55 (2013) 124039. 
[7] SCARABOSIO, A. et al., Journal of Nuclear Materials In press, corrected proof (2014) .

[8] KALLENBACH, A. et al., Nuclear Fusion 52 (2012) 122003.

[9] KALlENBACH, A. et al., Plasma Physics and Controlled Fusion 52 (2010) 055002.

[10] BEURSKENS, M. N. A. et al., Plasma Physics and Controlled Fusion 55 (2013) 124043.

[11] KUKUSHKIN, A. et al., J. Nucl. Mater. 438 (2013) S203.

[12] LIPSCHULTZ, B. et al., Fusion Science and Technology 51 (2007) 369.

[13] SCHAFFER, M. et al., Nucl. Fusion 37 (1997) 83.

[14] REIMOLD, F. et al., submitted to Nuclear Fusion (2014).

[15] LEONARD, A. et al., Nucl. Fusion 52 (2012) 063015.

[16] BERNERT, M. et al., submitted to PPCF (EPS 2014) (2014).

[17] LOARTE, A. et al., Nucl. Fusion 38 (1998) 331.

[18] POTZEL, S. et al., Nuclear Fusion 54 (2014) 013001.

[19] POTZEL, S. et al., Journal of Nuclear Materials In press, corrected proof (2014) .

[20] LOARTE, A. et al., Plasma Physics and Controlled Fusion 45 (2003) 1549.

[21] GIROUD, C. et al., Nucl. Fusion 52 (2012) 063022.

[22] MADDISON, G. et al., Nucl. Fusion 54 (2014) 073016.

[23] MAGGI, C. et al., Nuclear Fusion 50 (2010) 025023 (20pp).

[24] SCHWEINZER, J. et al., Development of the $Q=10$ scenario for ITER on ASDEX Upgrade (AUG), EX/9-

4, paper presented at 25th IAEA Int. Conf. on Fusion Energy St Petersburg 2014.

[25] KALLENBACH, A. et al., Plasma Physics and Controlled Fusion 55 (2013) 124041. 